\title{
Towards Self-Powered Cameras
}

\author{
Shree K. Nayar Daniel C. Sims Mikhail Fridberg* \\ Department of Computer Science, Columbia University \\ New York, NY 10027, USA \\ nayarecs.columbia.edu
}

\begin{abstract}
We propose a simple pixel design, where the pixel's photodiode can be used to not only measure the incident light level, but also to convert the incident light into electrical energy. A sensor architecture is proposed where, during each image capture cycle, the pixels are used first to record and read out the image and then used to harvest energy and charge the sensors' power supply. We have conducted several experiments using off-the-shelf discrete components to validate the practical feasibility of our approach. We first developed a single pixel based on our design and used it to physically scan images of scenes. Next, we developed a fully self-powered camera that produces 30x40 images. The camera uses a supercap rather than an external source as its power supply. For a scene that is around 300 lux in brightness, the voltage across the supercap remains well above the minimum needed for the camera to indefinitely produce an image per second. For scenarios where scene brightness may vary dramatically, we present an adaptive algorithm that adjusts the framerate of the camera based on the voltage of the supercap and the brightness of the scene. Finally, we analyze the light gathering and harvesting properties of our design and explain why we believe it could lead to a fully self-powered solid-state image sensor that produces a useful resolution and framerate.
\end{abstract}

\section{Introduction}

We are in the midst of an imaging revolution. In the last year alone, roughly 2 billion digital imaging systems were sold worldwide [2], and over a trillion images are now on the Internet. In addition to photography, digital imaging is transforming fields as varied as entertainment, social networking, ecommerce, security and autonomous navigation. There is wide belief that we have only seen the tip of the iceberg. We are about to witness a second wave of the imaging revolution, one that promises to be deeper and broader in impact than the first. This wave is expected to transform diverse fields including wearable devices, inter-

\footnotetext{
${ }^{*}$ Mikhail Fridberg is the head of ADSP Consulting in Sharon, MA.
}

net of things, personalized medicine, smart environments, sensor networks and scientific imaging.

In many of the emerging applications of imaging, such as wearable devices and sensor networks, a major challenge will be to develop systems that can function for a very long duration (ideally, forever) without being externally powered. In the context of cameras, one approach would be to use a solar panel situated close to a camera to power the camera's electronics. This, however, would not only be expensive but also bulky. A compromise would be to create image sensors where only a portion of the sensor plane is used for sensing, while the remaining area is used for power generation. Such a sensor architecture has been suggested in the past [4][9] and shown to lower the external power consumption of the sensor. This approach, however, requires the sacrifice of valuable real-estate on the sensor plane for non-imaging purposes.

In our work, we take a more extreme approach. Our goal is to redesign the pixels of the sensor such that they not only measure incident light but can also harvest all the energy needed for the measurements to be read out. In our pixel design, we use a photodiode in the photovoltaic mode rather than the photoconductive mode used in conventional image sensors. This ensures that each pixel consumes zero power to measure light and yet can convert light into electrical energy for powering the readout of the image.

Rather than design a solid-state sensor to demonstrate our ideas, which would be a very expensive endeavor, we have chosen to develop our pixel array using off-the-shelf discrete components. At the core of our architecture is an array of compact photovoltaic cells that are commonly used for solar power generation. To test the light sensitivity of a photodiode in photovoltaic mode, we first implemented a single pixel and used a robot to scan a scene to create an image. We then constructed a $30 \times 40$ pixel array that is able to both measure an image and harvest energy during each image capture cycle. The sensor is powered by a supercap, which is charged during the harvesting period of each image capture cycle. A lens with an effective F-number of 3.5 is used to form images on the sensor array. We have conducted several experiments to test the power performance 
of the camera. We show that for an indoor scene that is well lit (around 300 lux) the camera is able to produce one image per second, indefinitely. We discuss how such a system can adjust its framerate based on scene brightness, which directly impacts the amount of harvested energy.

By measuring the power generated by our sensor array, we estimate the power that would be generated by a camera that uses a compact solid-state image sensor. We then use the power specification of a commercially available image sensor to determine that, for a scene that is approximately 300 lux in brightness, a compact camera that uses a solidstate sensor based on our design would be able to produce at least a 200x200 image per second. Since the power generation and power consumption estimates we use in our calculations are conservative, we can expect an optimized solidstate sensor based on our design to produce an appreciably higher resolution and/or framerate.

\section{Related Work}

In recent years, we have seen a few attempts at developing image sensors that both sense images and harvest energy. Fish et al. [3] proposed a design where each pixel includes two photodiodes, one used for sensing and the other for harvesting. Shi et al. [9] also proposed a twophotodiode pixel design, but with the difference that the sensing photodiode also contributes to harvesting. One disadvantage of this design is that it requires a large number of transistors (about 18) per pixel. Although both sensing and harvesting capabilities are incorporated into each pixel in the above designs, a large fraction of the pixel area must be sacrificed for harvesting. In [4], the image sensor area itself is split into two, one with an array of photodiodes for sensing and another with an array for harvesting.

In work that is closer to ours, Law et al. [7] use only one photodiode in each pixel and a set of 6 transistors to switch between sensing and harvesting modes. In this design, each pixel needs to be powered during sensing as the photodiode is operated in photoconductive mode. In contrast, we operate the photodiode in photovoltaic mode and hence the pixel uses zero power for sensing. Furthermore, our design is significantly simpler, requiring only two transistors per pixel. Ay [1] has demonstrated an alternative design in which each pixel has two photodiodes where one is used for harvesting but both are used for sensing. The disadvantage in this case is that only half the image sensor area is used for harvesting. Unlike most of the other attempts, Ay has implemented a 54x50 pixel low-powered image sensor and used it to capture images of real scenes.

There are several significant differences between previous work and ours: (a) While previous pixel designs have used the photodiode in photoconductive mode, where the pixel needs to be powered at all times and produces dark current even when it is not illuminated, we operate the diode in photovoltaic mode which uses zero power to measure image irradiance and does not produce dark current. Although the response time of the photodiode is slower in photovoltaic mode, we have found it to be fast enough (a few milliseconds) to capture dynamic scenes that are reasonably well lit. Furthermore, as shown recently by Ni [8], using the photodiode in photovoltaic mode has the advantage that the sensor can capture a much wider dynamic range. (b) Whereas most previous designs dedicate a part of the sensor area for harvesting alone, in our case each pixel can both measure light and harvest energy. (c) Finally, while previous work seeks to enhance the power performance of CMOS sensors, our goal is to develop fully self-powered imaging systems. At the end of the paper, we provide calculations that show that our approach could lead to compact solid-state image sensors that provide meaningful resolution and framerate while being fully self-powered.

\section{Architecture for Self-Powered Image Sensor}

We begin by explaining the working principle of a photodiode, and describe a pixel design that is commonly used in CMOS image sensors. Then, we describe the design of our self-powered pixel and a variant that measures the time to achieve a preset voltage. Finally, we show the architecture of our complete image sensor which includes a power harvester and a readout circuit.

\subsection{Photodiode Model}

At the heart of each pixel in any camera is the photodiode, the internal model of which is shown in Figure 1. It is a P-N junction semiconductor which acts like an ordinary diode $D$, with a capacitance $C$, shunt resistance $R_{s h}$ and series resistance $R_{s e}$. However, unlike a regular diode, the photodiode generates current when exposed to light. When an external load is connected to it, the current flows from its anode through the load and back to its cathode. The total current through the photodiode is the sum of the photocurrent $I_{p d}$ (due to light) and the dark current $I_{d}$ (without light).

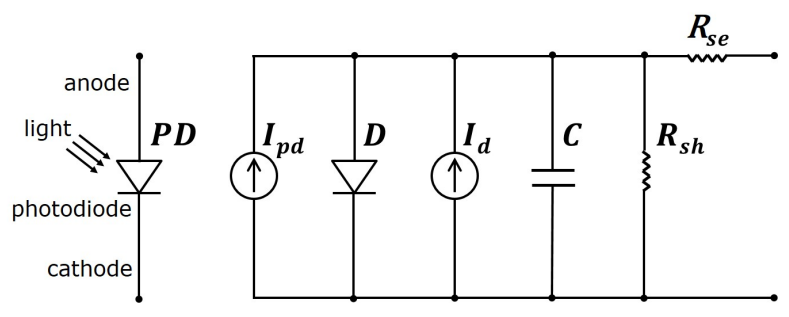

Figure 1. Model of a photodiode. (Adapted from [10].) 


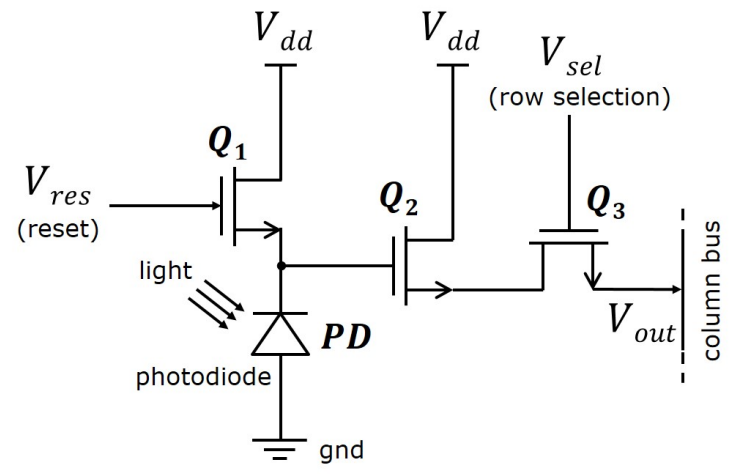

Figure 2. Pixel structure commonly used in image sensors.

\subsection{Conventional Pixel Design}

Figure 2 shows the structure of a pixel that is commonly used in conventional image sensors [11] ${ }^{1}$. In this case, the photodiode $P D$ is reverse-biased (in photoconductive mode) with the anode connected to ground and the cathode connected to a voltage $V_{d d}$ through the transistor $Q_{1}$. First, $P D$ is reset by applying voltage $V_{\text {res }}$ to $Q_{1}$. This brings the voltage of the cathode to $V_{d d}$. When light is incident on $P D$, current begins to flow through it and the voltage across it drops by an amount that is proportional to the incident light energy and the exposure time. This voltage is buffered by source follower transistor $Q_{2}$, and is read out as $V_{\text {out }}$ when the pixel is selected by applying $V_{\text {sel }}$ to transistor $Q_{3}$. In this design, $P D$ consumes power during reset and $Q_{2}$ is powered during readout. In addition, since $P D$ is reverse-biased, its output voltage is affected not only by the incident light but also by dark current, which, although very small, exists even when there is no light.

\subsection{Self-Powered Pixel Design}

Figure 3 shows the pixel design we use in our sensor. In this case, the photodiode $P D$ is operated in photovoltaic mode with zero bias. It is reset by applying voltage $V_{\text {res }}$ to transistor $Q_{1}$. Subsequently, the voltage of the anode of $P D$ increases to a level proportionate to the incident light energy. $V_{\text {out }}$ is read out by applying $V_{\text {sel }}$ to transistor $Q_{2}$. Note that in this case $P D$ draws zero power to produce a voltage proportionate to the incident light, and since it is not biased it does not produce any dark current. An important feature of the design is that $V_{\text {res }}$ can be applied to $Q_{1}$ to not only reset $P D$ but to also harvest energy. That is, the emitter of transistor $Q 1$ can be switched between ground (for resetting) and a power supply (for harvesting).

Note that the above design is as simple as can be, requir-

\footnotetext{
${ }^{1}$ Both 3-transistor (3T) and 4-transistor (4T) designs are widely used today (see [5]). For our purposes here, it suffices to understand the working principle of either one.
}

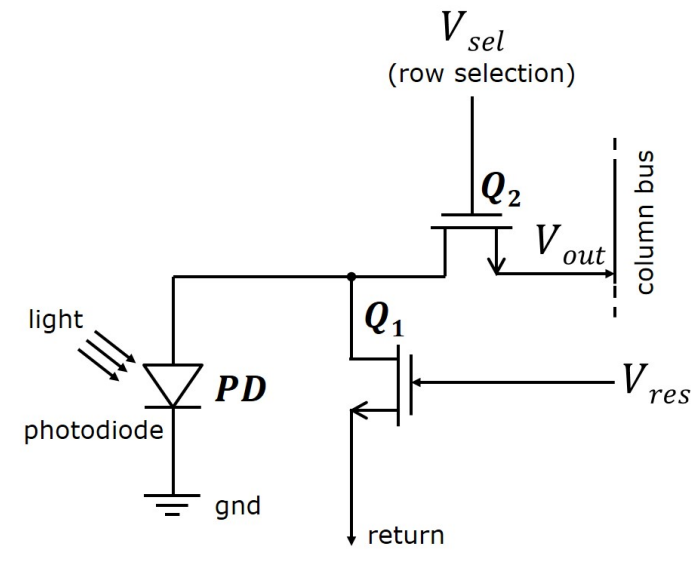

Figure 3. Proposed pixel design for sensing light and harvesting energy.

ing the use of just a single photodiode and two transistors. Furthermore, as mentioned earlier, the use of the photodiode in photovoltaic mode has the advantage of measuring a wider dynamic range [8]. The one disadvantage of our design compared to the conventional one shown in Figure 2 is that, because $P D$ is not reverse biased, its response to light is slower. In our experience, however, we are able to capture images of well-lit indoor scenes in a few milliseconds using the photovoltaic mode. In any case, we require the scene to produce reasonable light levels for the sensor to be self-powered.

An alternative approach to measuring the incident light energy using the photovoltaic mode is to measure the time it takes for the diode voltage to reach a predefined threshold voltage $V_{\text {thresh }}$. In this case, the pixels function in an asynchronous fashion as each one is read out only when it reaches $V_{\text {thresh }}$. Figure 4 shows how a photodiode in photovoltaic mode can be used to measure time-to-voltage. The capacitor $C$ is used to smooth the voltage of $P D$ as well as control the speed of integration, and the comparator $C P$ is

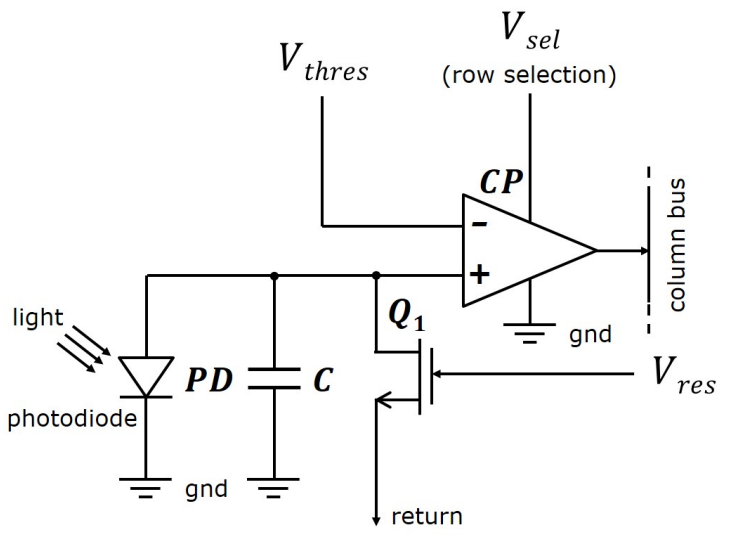

Figure 4. Alternative pixel structure used to measure time-tovoltage. 


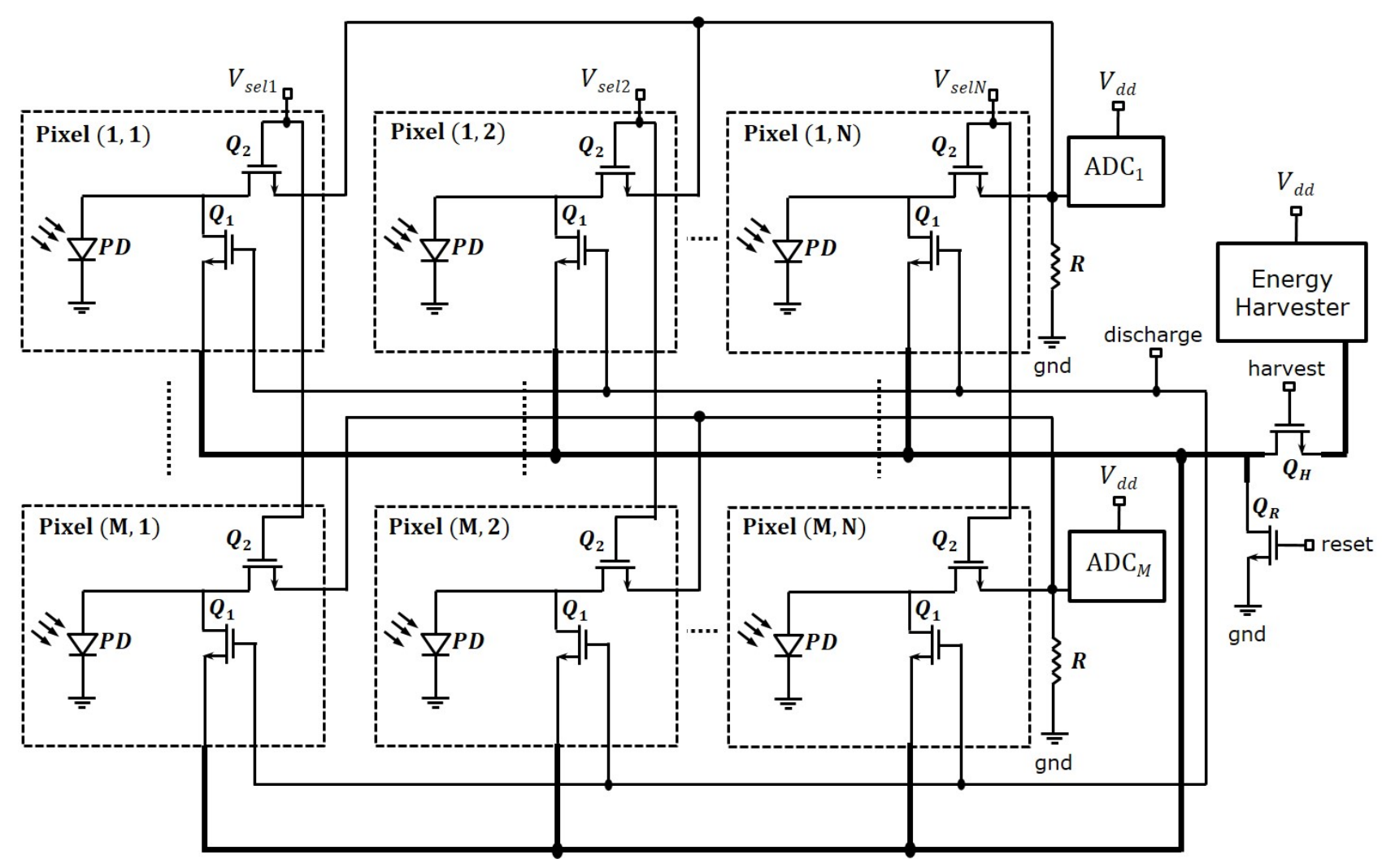

Figure 5. Architecture of the self-powered image sensor.

used to detect when the voltage reaches $V_{\text {thresh }}$. In this case, due to the use of a comparator, the pixel does need to be powered at all times. Although we do not use this design to implement our pixel array, we do use it to implement a single pixel to test the sensitivity of the photovoltaic mode.

\subsection{Self-Powered Image Sensor Architecture}

Figure 5 shows the architecture of our self-powered image sensor. Each pixel has the structure shown in Figure 3 . In addition to the $2 \mathrm{D}$ array of pixels, the sensor includes a harvesting power supply, a microcontroller, an analog-todigital convertor (ADC) for each sensor row, and transistors $Q_{R}$ and $Q_{H}$ for resetting all the pixels and harvesting energy from all the pixels, respectively. The microcontroller is not shown in the figure, but is programmed to control the ADCs and to generate the signals to transistors $Q_{2}$ to select pixels during readout, to all $Q_{1}$ transistors to discharge, to $Q_{R}$ for resetting and to $Q_{H}$ for harvesting.

The control of the image sensor works in the following way. First, a pulse is applied to the discharge and reset pins for a duration $T_{\text {res }}$ to reset all the pixels. Then, the pixels are allowed to "integrate" for time $T_{\text {int }}$. Next, $V_{\text {sel }}$ is applied to each column (starting with the leftmost one) of pixels and the voltages $V_{\text {out }}$ of these pixels are read out using the ADCs. This column-wise readout of the entire array takes time $T_{\text {read }}$. Finally, signals are applied for a duration $T_{\text {harv }}$ to the discharge and harvest pins, so that current flows from all pixels ${ }^{2}$ on the sensor through the harvesting power supply. Thus, the total time taken to capture an image is:

$$
T_{\text {image }}=T_{\text {res }}+T_{\text {int }}+T_{\text {read }}+T_{\text {harv }} .
$$

The framerate of the self-powered camera is $R=$ $1 / T_{\text {image }}$, and we can define its duty cycle as $D=$ $100\left(T_{\text {image }}-T_{\text {harv }}\right) / T_{\text {image }} \%$.

Note that the energy harvested from the pixels includes the energy accumulated during integration and also that generated during the harvesting period. The harvested energy must be stored in some device. This can either be a small rechargeable battery or a supercap. If a supercap is used, it would have to be charged by an external source before the camera can begin to function. Such an external charge-up would also be needed if the supercap gets depleted because the camera is shown a dark scene for a long period. For these reasons, it would be more convenient to use a rechargeable battery. As we shall see later, the sen-

\footnotetext{
${ }^{2}$ Note that the pixels will have different voltages as they are exposed to different scene radiances. Therefore, to maximize energy harvesting, it would be better to discharge the pixels one at a time. However, this would require a more sophisticated harvesting strategy which in turn might increase power consumption. This is an important issue that deserves further exploration.
} 


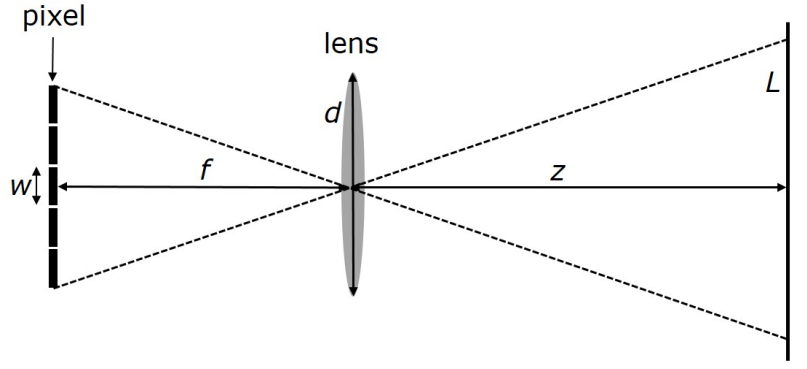

Figure 6. Image formation using a lens.

sor can be operated with a scene-adaptive framerate, where $T_{\text {harv }}$ is continuously adjusted based on the voltage of the power supply and the brightness of the scene.

\section{Image Formation and Incident Energy}

The energy harvested by our image sensor is proportional to the total light flux received by the sensor. We now describe how this flux is related to scene radiance and the optics used to form an image. Consider the lens-based imaging system shown in Figure 6. If the diameter of the lens is $d$ and the effective focal length of the system is $f$, image irradiance is related to object radiance $L$ as [6]:

$$
E=L \frac{\pi}{4}\left(\frac{d}{f}\right)^{2} \cos ^{4} \alpha,
$$

where, $\alpha$ is the view angle with respect to the optical axis. The ratio $N=f / d$ is the effective F-number of the imaging system, and the irradiance is inversely proportional to $N^{2}$.

It is easy to compare the light efficiency of a large system such as ours with that of a compact system that uses a solidstate image sensor. If the effective F-numbers of the two systems are $N_{1}$ and $N_{2}$, respectively, the ratio of the image irradiances in the small and large systems for any given scene radiance is:

$$
\frac{E_{2}}{E_{1}}=\frac{N_{1}^{2}}{N_{2}^{2}} \text {. }
$$

Since for a fixed field of view the ratio of the areas of the small and large image sensors is $A_{2} / A_{1}={f_{2}}^{2} / f_{1}{ }^{2}$, the ratios of the total flux received by the two sensors is:

$$
\frac{\phi_{2}}{\phi_{1}}=\frac{N_{1}^{2}}{N_{2}^{2}} \cdot \frac{f_{2}^{2}}{f_{1}^{2}}=\frac{d_{2}^{2}}{d_{1}^{2}} .
$$

In short, the ratio of the energies harvested by the two systems is simply the ratio of the areas of their lens apertures. Since the compact system is expected to have a smaller lens aperture, it will generate less power. However, as we shall see in Section 7, a typical solid-state image sensor consumes significantly less power per pixel than our large format system, and this offsets the drop in harvested energy.

\section{Power Adaptive Framerate}

When it is in fully self-powered mode, we can adjust the amount of time $T_{\text {harv }}$ the sensor harvests energy during each image capture cycle, based on the power available in the supply. A simple approach would be to adjust $T_{h a r v}$ as a function of the difference $V_{\text {diff }}$ between a desired setpoint $V_{d}$ and the current voltage $V(t)$ of the supply. However, the voltage of the supply alone would only be a coarse measure of how much power remains in the supply, since the voltage would vary gradually as a function of stored power. For more fine-grained control we need to also vary $T_{\text {harv }}$ based on the brightness of the scene being imaged. This is done by making $T_{h a r v}$ a function of the difference $I_{\text {diff }}$ between a setpoint $I_{d}$ that represents the current used by the sensor during a readout cycle and the current $I(t)$ that flows into the supply at the beginning of the harvesting period. If $T_{o}$ is the harvesting time needed for the sensor to be fully self-powered for a scene of normal brightness, the actual harvesting time $T_{\text {harv }}$ can be adapted as:

$$
T_{\text {harv }}(t)=T_{o}+\alpha V_{\text {diff }}(t)+\beta I_{\text {diff }}(t),
$$

where, $\alpha$ and $\beta$ are preset weights related to the voltage and current differences, respectively. In effect, the above is a proportional $(\mathrm{P})$ controller. More sophisticated adaptation can be achieved using a proportional-integral-derivative (PID) controller which would also use the time derivatives and integrals of $V_{d i f f}(t)$ and $I_{d i f f}(t)$. In any of these adaptive modes, the camera can run indefinitely as it produces each image only when it can "afford" to.

\section{Experiments}

We have conducted a series of experiments to validate the ideas proposed in this paper. The photodiodes used in all our experiments (BPW34 from Vishay Semiconductors) are operated in the photovoltaic mode. To ensure that this mode would have the sensitivity needed to measure images of real-world scenes, we first developed the single pixel system shown in Figure 7(a), based on the time-to-voltage design shown in Figure 4. The active area of the photodiode is about $2.8 \mathrm{~mm} \times 2.8 \mathrm{~mm}$. For the optics of the pixel, we use a straw that is $4 \mathrm{~mm} \times 4 \mathrm{~mm}$ in cross-section and 250 $\mathrm{mm}$ long ${ }^{3}$. A microcontroller (MC13226V Freescale Semiconductor) is used to reset the photodiode and to measure the time it takes to reach a preset voltage. In this timeto-voltage implementation, the total time taken to make a single pixel measurement was set to $100 \mathrm{msec}$.

The radiometric response curve of the pixel, shown in Figure 7(b), was measured by pointing it at a display whose brightness was increased from 0 to 255 in increments of 1 , and recording the time-to-voltage for each brightness. Since

\footnotetext{
${ }^{3}$ The straw and all other opto-mechanical parts used in our experiments were 3D printed using a Makerbot Replicator and a Stratasys uPrint SE.
} 


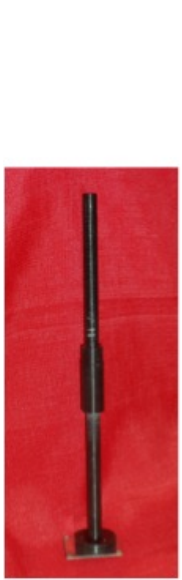

(a)

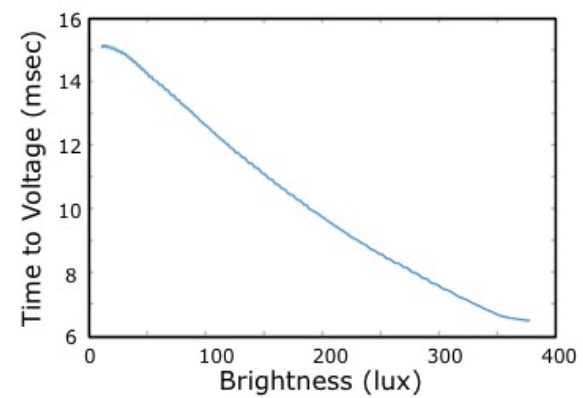

(b)

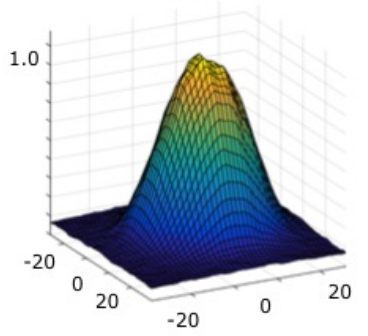

(c)

Figure 7. (a) Single pixel with photodiode in photovoltaic mode attached to a long straw. (b) Measured radiometric response curve of the pixel. (c) Measured PSF of the pixel for a depth of $94.6 \mathrm{~cm}$.

the display itself is expected to have a non-linear radiometric response, we measured its true brightness (in lux) by using a light meter placed next to the straw opening. Since the photodiode is operated in photovoltaic mode, as expected, the radiometric response function is non-linear with larger brightness values being more compressed [8]. We next measured the point spread function (PSF) of the pixel by raster scanning a small white spot across the display, and recording a pixel measurement for each spot location. These measurements were linearized using the response curve to get the PSF shown in Figure 7c. In the case of a straw, the width of the PSF scales linearly with distance from the straw.

We attached the pixel to the end-effector of an Adept Model 840 robot to scan an image of the scene, as shown in Figure 8(a). The pixel was moved in steps of $2 \mathrm{~mm}$ along both dimensions of a plane to capture the 250x115 image shown in Figure 8(b). Due to the PSF of the straw, the image is blurred, with the degree of blur increasing with depth (the fruits in the back are more blurred than the mannequin). We used interpolation to upsample the image by a factor of 4 in each dimension, and then deblurred it with the PSF of the straw (with the PSF scale chosen for the depth of the mannequin) using the Lucy-Richardson algorithm.

The above single-pixel experiment demonstrated that the photovoltaic mode has the response and sensitivity needed to capture high quality images at video rate. Although the time-to-voltage design has the advantage that it can yield very wide dynamic range, it requires the image sensor to read out pixels asynchronously, which requires a more complex readout circuit. Therefore, we decided to implement

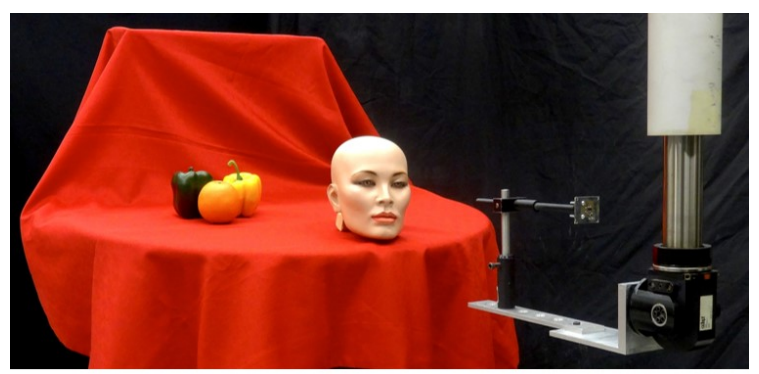

(a)

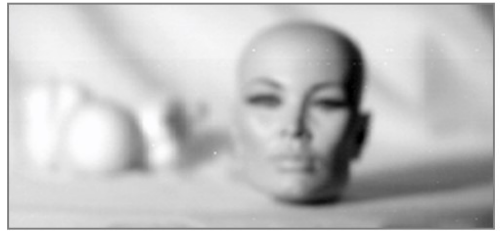

(b)

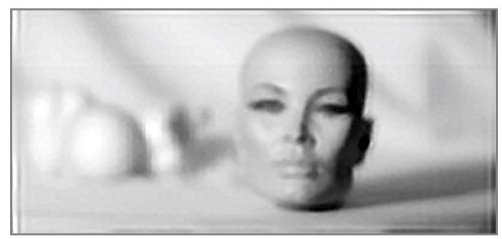

(c)

Figure 8. (a) A scene is scanned by the pixel (Figure 7(a)) using a robot. (b) Image captured by the scanning process, where the pixel brightness values are linearized with respect to scene radiance using the response curve shown in Figure 7(b). (c) Image obtained by deconvolving the captured image with the pixel's PSF shown in Figure 7(c).

our sensor array based on the pixel design shown in Figure 3 and the architecture shown in Figure 5. The array, shown in Figure 9(a), has 30x40 photodiodes (BPW34 from Vishay Semiconductors) each approximately $4 \mathrm{~mm} \times 4 \mathrm{~mm}$ in size with an active area of $2.8 \mathrm{~mm} \times 2.8 \mathrm{~mm}$. Since the photodiodes have leads on two of their sides, each one was oriented at 45 degrees so as to achieve a low pixel pitch of $7 \mathrm{~mm}$. The entire array covers a sensing array of $210 \mathrm{~mm} x$ $280 \mathrm{~mm}$. Also seen on the front of the board are the readout transistors and the microcontroller (STM32L151ZDT6 from ST Microelectronics).

On the back side of the circuit board is an array of twoswitch packages (NLAS4717MR2G from On Semiconductor), each package including the two transistors used in the corresponding pixel. The back side also includes the global reset and harvest switches and the harvesting power supply (BQ25504RGT16 from Texas Instruments). Note that the board does not have a battery but instead a $0.5 \mathrm{~F}$ supercap, which is charged to start the camera but is recharged using just energy harvested from the pixels. As seen in Figure 9(b), a single Fresnel lens (32-685 from Edmund Optics) is used to form the image. The system has a effective focal 


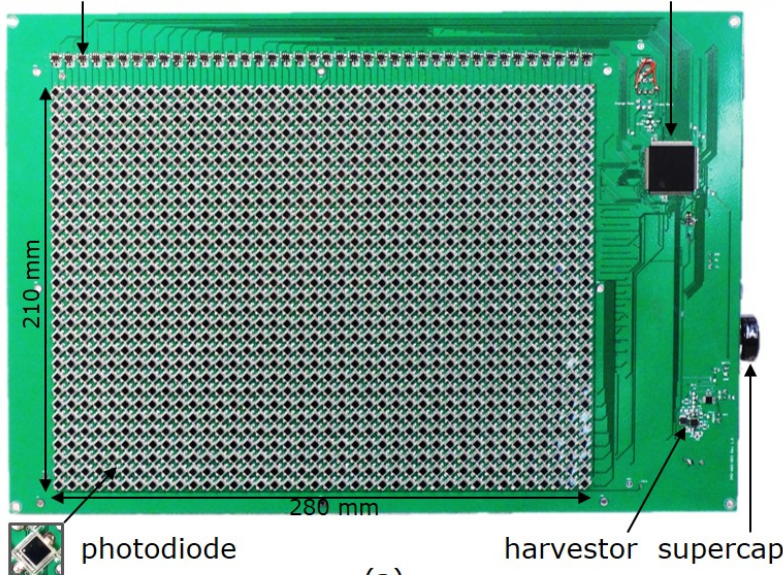

(a)

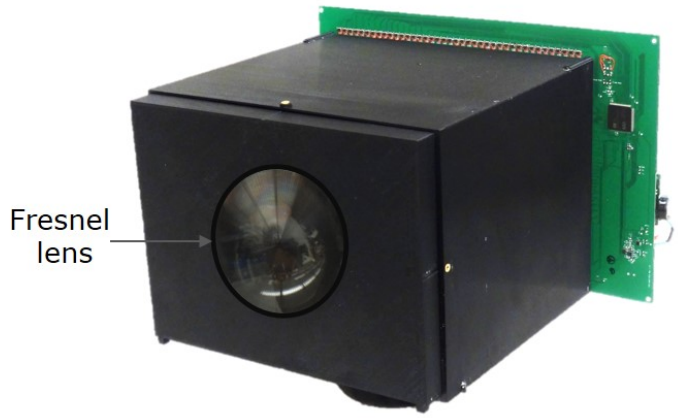

(b)

Figure 9. A self-powered camera with 30x40 pixels. (a) The front of the printed circuit board includes an array of photovoltaic cells, the readout transistors and the microcontroller. The back of the board includes the discrete components of each pixel and the energy harvester. For storage of harvested energy, a supercap is used instead of a battery. (c) A Fresnel lens with an effective F-number of 3.5 is used to form an image on the pixel array.

length of $375 \mathrm{~mm}$ and clear aperture diameter of $127 \mathrm{~mm}$, resulting in an effective F-number $N=3.5$.

When the voltage across its supercap is above $2.5 \mathrm{~V}$, the camera automatically starts capturing 30x40 images. Manual switches are included to control the exposure time and the framerate. In most of our experiments, we use an exposure time of $15 \mathrm{msec}$ and a framerate of 1 image per second. Since we have used off-the-shelf components, the pixels differ slightly in terms of their response functions and gains. To address this issue, we performed a calibration where the camera was shown a flat white board with uniform illumination. The average brightness of the board was varied from 0 lux to 2500 lux in steps of 50 lux and an image was captured for each brightness level. This data was linearly interpolated to create a dense lookup table for each pixel that maps its measured voltage to scene brightness. The response function of one of the pixels is shown in Figure 10. As can be seen the response is non-linear, enabling each pixel to cap-

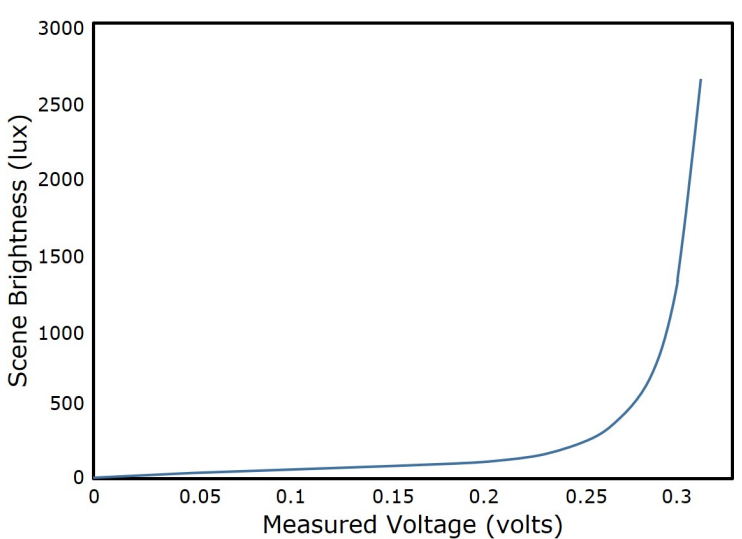

Figure 10. The radiometric response of a single pixel (with exposure time of $15 \mathrm{msec}$ ) showing the relation between measured pixel voltage and scene brightness. The response function is nonlinear as the photodiode is operated in photovoltaic mode, enabling the pixel to measure a wide dynamic range [8].

ture a wide dynamic range.

Each captured image is transferred to a computer via a serial link $^{4}$, where the measured pixel voltages are first mapped to scene brightness and then a scale and gamma correction is applied to the image for the purpose of display. The meta tag of each image includes the various parameters (exposure, harvesting time, etc.) used to capture the image as well as the voltage across the supercap. Figure 11 shows a video sequence (30x40 pixels at 1 frame per second) captured by the camera in self-powered mode.

Figure 12 shows the setup we used to evaluate the power performance of the camera. The brightness of the scene is controlled by varying the intensity of the light source using a dimmer. The scene brightness was measured using a light meter placed right next to the lens of the camera and facing the scene. We started-up the camera by connecting its supercap to an external power source, set its voltage to $2.74 \mathrm{~V}$, and then disconnecting the source. Each second, an image of the scene is recorded along with the voltage across the supercap.

The plot shown in Figure 13 shows scene brightness (in orange) and supercap voltage (in blue) plotted as a function of time over a period of 80 minutes. As the orange plot shows, the scene brightness was ramped from 150 lux to 1150 lux over a period of $20 \mathrm{~min}$, kept constant at 0 lux for the next $20 \mathrm{~min}$, stepped up to 1000 lux for the next $12 \mathrm{~min}$, and dropped to 200 lux for the last $28 \mathrm{~min}$. The blue plot shows the voltage across the supercap as a function of time. Note that without energy harvesting the supercap voltage would quickly fall until the camera stopped functioning. Due to harvesting, the supercap voltage increases and decreases with the scene brightness, and at a

\footnotetext{
${ }^{4}$ In some applications, we would want the camera to be an untethered, standalone device. In such cases, we would need to use a portion of the harvested power to wirelessly communicate images to a remote location.
} 

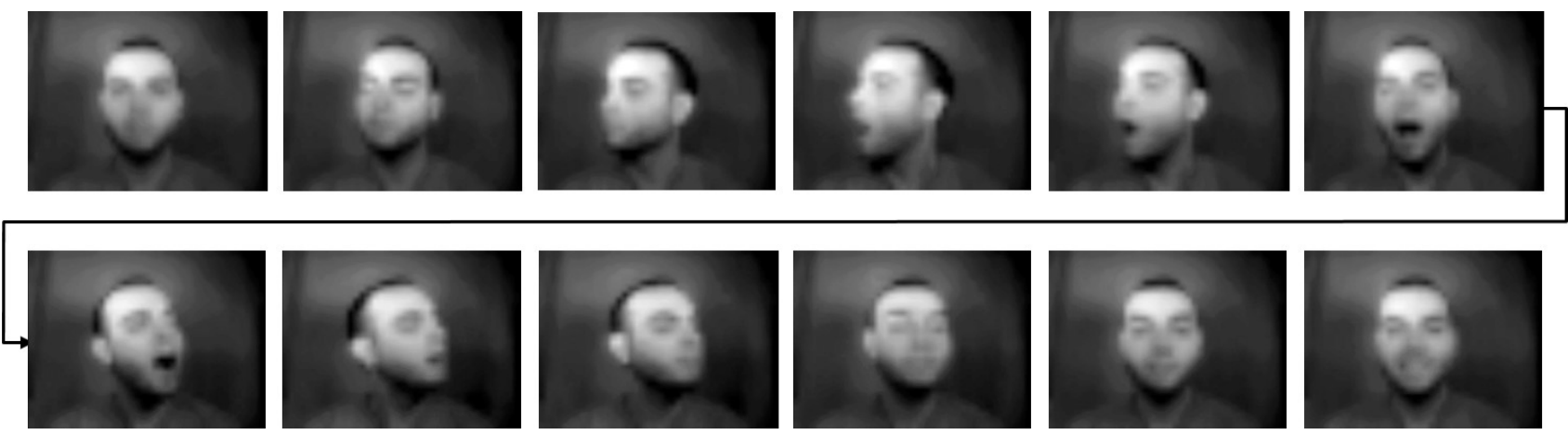

Figure 11. A video sequence captured by the self-powered camera. Each image has 30x40 pixels and the framerate is 1 image per second.

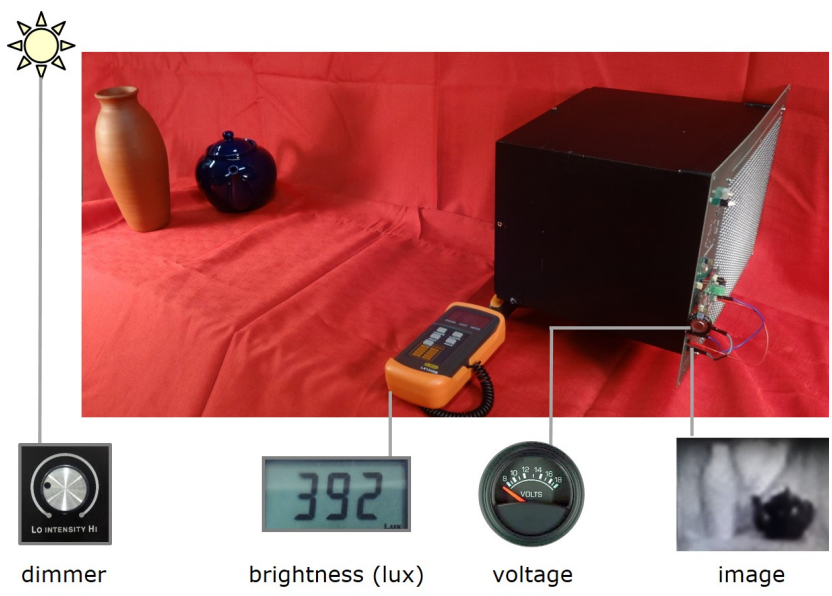

Figure 12. The setup used to evaluate the power performance of the camera.

steady brightness of 200 lux the voltage stabilizes at around $3 \mathrm{~V}$, which is well above the minimum of $2.5 \mathrm{~V}$ needed for the camera to function. Since the exposure time is $15 \mathrm{msec}$ and image readout takes $18 \mathrm{msec}$, we can say that the duty cycle of the camera for a scene that is 200 lux in brightness is $D=100(0.015+0.018) / 1=3.3 \%$. While this is an empirically determined duty cycle, in Appendix A we show how the duty cycle of any self-powered camera can be analytically modeled. The use of such a model, however, requires detailed knowledge of the specifications of all of its components.

Figure 14 shows a few images from a video of a rotating mannequin. A black board is placed on one side of the mannequin to increase the variability in scene brightness as the mannequin rotates. Shown in each image is the voltage of the supercap, where the colors red and green are used to represent a decrease and increase in voltage, respectively, with respect to the previous frame.

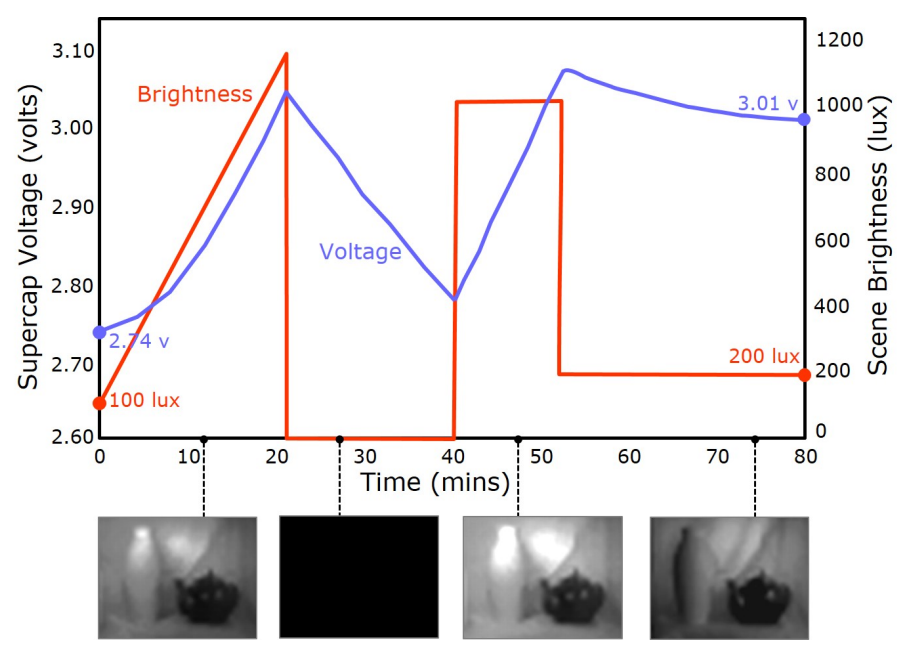

Figure 13. The camera is powered solely by its supercap over a period of 80 minutes, without the use of an external power source. Since the pixels not only record images but also harvest energy and charge the supercap, the voltage across the supercap varies with scene brightness. At 200 lux, the voltage stabilizes at around $3 \mathrm{~V}$, above the minimum voltage needed for the camera to function.

\section{A Fully Self-Powered Solid-State Sensor?}

We have done some rough calculations to determine the resolution and framerate one could get from a self-powered CMOS image sensor based on our architecture. Although far from optimized, we used our current prototype to estimate the harvesting capability of the design. First, we imaged a scene with a vase (to ensure the scene was spatially varying in brightness) and measured the power generated by the array for different scene brightness values (from 200 lux to $700 \mathrm{lux}$ ) and for different load resistors (from $10 \mathrm{Ohms}$ to $80 \mathrm{Ohms}$ ) connected to the array. We varied the load because energy harvesters do exactly that to maximize the harvested energy. The plot in Figure 15 shows the result. If we pick a reasonable scene brightness of 300 lux (welllit indoor scene) we see that the array produces $1.1 \mathrm{~mW}$ 

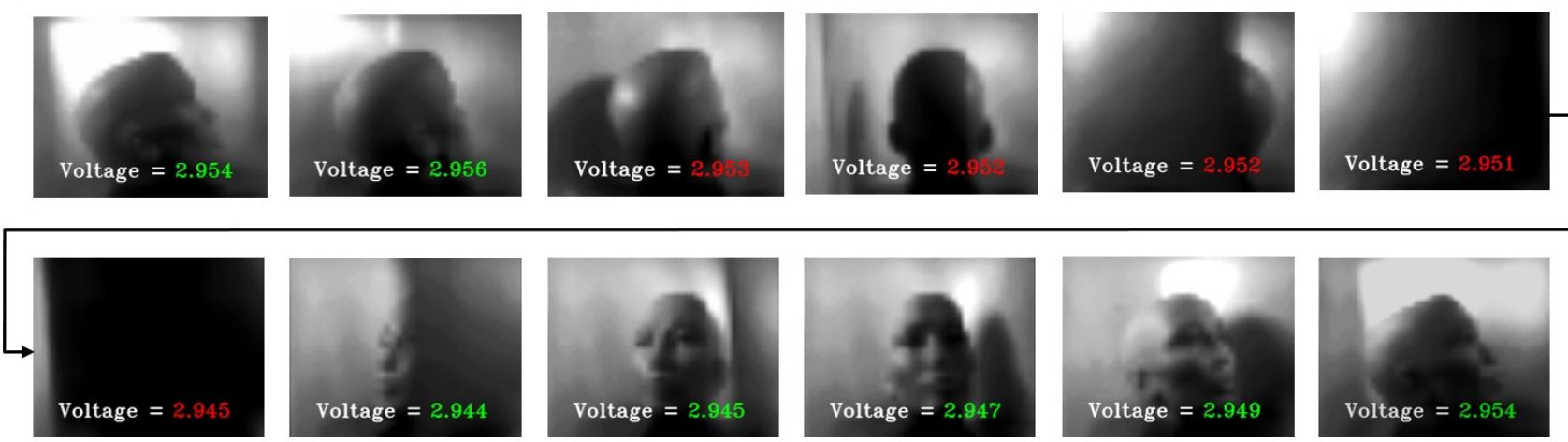

Figure 14. A video sequence of a rotating mannequin with a black board placed on one of its sides. Each image includes the voltage across the supercap, which is shown in red when it drops and green when it rises.

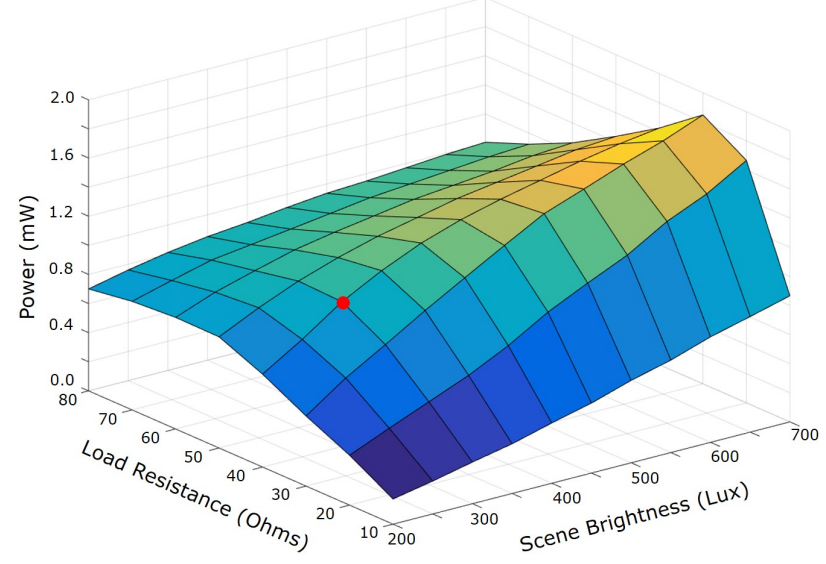

Figure 15. The power generation capability of our 30x40 array was measured by connecting several load resistors to the array, for different scene brightness values. This plot shows that an adaptive energy harvester would draw $1.1 \mathrm{~mW}$ (red dot) from the array for a scene brightness of 300 lux (indoor lighting).

(red dot shown on the plot). If we assume the harvester has an efficiency of $70 \%$, the harvested power would be $P_{1}$ $=0.77 \mathrm{~mW}$. This power is generated by a sensing area $A_{1}$ $=2.8 \times 2.8 \times 30 \times 40 \mathrm{~mm}^{2}=9408 \mathrm{~mm}^{2}$, using a lens with Fnumber $N_{1}=3.5$.

Now, let us replace our sensor with a $1 / 2$ inch solid-state sensor with area $A_{2}=8.8 \times 6.6 \mathrm{~mm}^{2}=58.08 \mathrm{~mm}^{2}$, and replace our lens with a faster one with effective F-number $N_{2}=1.4$. Then, using equations 3 and 4 , we can determine the power generated by the solid-state sensor to be $P_{2}$ $=\left(P_{1} A_{2} N_{1}^{2}\right) /\left(A_{1} N_{2}^{2}\right)=0.0297 \mathrm{~mW}$.

To find the resolution and framerate that $P_{2}$ can yield in the case of a typical CMOS image sensor, we use the power specifications of a commercially available image sensor. Since it is power optimized we chose Omnivision's OV2740 sensor, which uses $0.7 \mathrm{~nJ}$ to produce a single pixel measurement. If such a sensor had both sensing and har- vesting capabilities, we could use the $0.0297 \mathrm{~mW}$ harvested by it to measure and read out $0.0297 / 0.0075=42,428$ pixels per second. This is roughly a $210 \times 200$ pixel image per second, or a lower resolution image at a higher framerate, or a higher resolution image at a lower framerate. Note that this calculation is conservative as it uses the power generation performance of our unoptimized sensor and the power consumption estimate of a commercially available sensor with several additional functionalities. It is quite possible that a highly power-optimized design can result in a fully self-powered camera with an appreciably higher resolutionframerate product.

\section{A. Analytical Model for the Duty Cycle}

When we have detailed information regarding all the optical and electronic components of a self-powered imaging system, it is possible to analytically derive the duty cycle of the system. Let us assume that the brightness of the scene is $E_{v}$ lux. This quantity can be converted to scene radiance as $L=E_{v} / 683 \mathrm{Watts} / \mathrm{str} / \mathrm{m}^{2}$. If a lens with F-number $N$ is used, the image irradiance can be determined using equation 2 as $E=L \pi /\left(4 N^{2}\right)$ Watts $/ \mathrm{m}^{2}$. Note that this estimate of irradiance does not include attenuations due to absorption and reflection by the lens, the $\cos ^{4} \alpha$ spatial fall-off in equation 2 , or other effects such as vignetting. These can be bundled into a single optical efficiency factor $O_{e}$ to determine the actual image irradiance as $E_{i}=E O_{e}$ Watts $/ \mathrm{m}^{2}$. If the image sensor has pixels with linear dimension $w$, a $100 \%$ fill-factor, and $n_{h}$ and $n_{v}$ pixels in the horizontal and vertical directions, the total flux received by the sensor is $\phi=E_{i} w^{2} n_{h} n_{v}$ Watts.

Now let us assume that the complete harvesting pipeline of the camera has a conversion efficiency $H_{e}$. Then, the power produced due to harvesting is $P_{h}=\phi H_{e}$ Watts. Note that we have assumed a monochrome sensor here. If we use a color mosaic, such as the Bayer pattern, to capture three color channels, we would reduce the harvested 
power by roughly a factor of 3 . If the power consumed by the camera to capture a single image is $P_{i}$, the duty cycle of the camera is $D=100 P_{h} /\left(P_{h}+P_{i}\right) \%$.

\section{Acknowledgements}

This research was conducted in the Computer Vision Laboratory at Columbia University. It was supported by ONR Grant No. N00014-14-1-0741. The authors are grateful to Behzad Kamgar-Parsi at ONR for his support of the project. The authors thank Eric Fossum of Dartmouth College for his detailed technical feedback on the paper. Mohit Gupta and Anne Fleming proofread the paper; Xiaotang Wang, Wei Wang and Divyansh Agarwal helped with image processing; and Bill Miller helped with 3D modeling and printing.

\section{References}

[1] S. U. Ay. A CMOS Energy Harvesting and Imaging (EHI) Active Pixel Sensor (APS) Imager for Retinal Prosthesis. IEEE Trans. on Biomed. Circuits and Systems, 5(6):535-545, 2011.

[2] C. Chute. Worldwide Digital Camera 2014-2018 Forecast Update. International Data Corporation, Oct. 2014.

[3] A. Fish, S. Hamami, and O. Yadid-Pecht. Self-powered active pixel sensors for ultra low-power applications. IEEE Inter. Symp. on Circuits and Systems, 5:5310-5313, May 2005.

[4] A. Fish, S. Hamami, and O. Yadid-Pecht. CMOS image sensors with self-powered generation capability. IEEE Trans. on Circuits and Systems II: Express Briefs, 53(11):1210-1214, Nov. 2006.

[5] E. R. Fossum and D. B. Hongdongwa. A Review of the Pinned Photodiode for CCD and CMOS Image Sensors. IEEE Journal of the Electronic Devices Society, 2(3):33-43, May 2014.

[6] B. K. P. Horn. Robot Vision. MIT Press, 1986.

[7] M. K. Law, A. Bermak, and C. Shi. A low-power energyharvesting logarithmic CMOS image sensor with reconfigurable resolution using two-level quantization scheme. IEEE Trans. on Circuits and Systems II: Express Briefs, 58(2):8084, Feb. 2011.

[8] Y. Ni. 1280x1024 logarithmic snapshot image sensor with photodiode in solar cell mode. International Image Sensor Workshop, June 2013.

[9] C. Shi, M. Law, and A. Bermak. Novel Asynchronous Pixel for an Energy Harvesting CMOS Image Sensor. IEEE Trans. on Very Large Scale Integ. (VLSI) Syst., 19(1):118129, 2011.

[10] ThorLabs. Photodiode: Theory of Operation. https://www.thorlabs.com/tutorials.cfm? tabID $=31760$.

[11] S. Vargas-Sierra, E. Roca, and G. Lin-Cembrano. Pixel Design and Evaluation in CMOS Image Sensor Technology. Proc. of XXIV Conf. on Design of Circuits and Integ. Syst., 2009. 